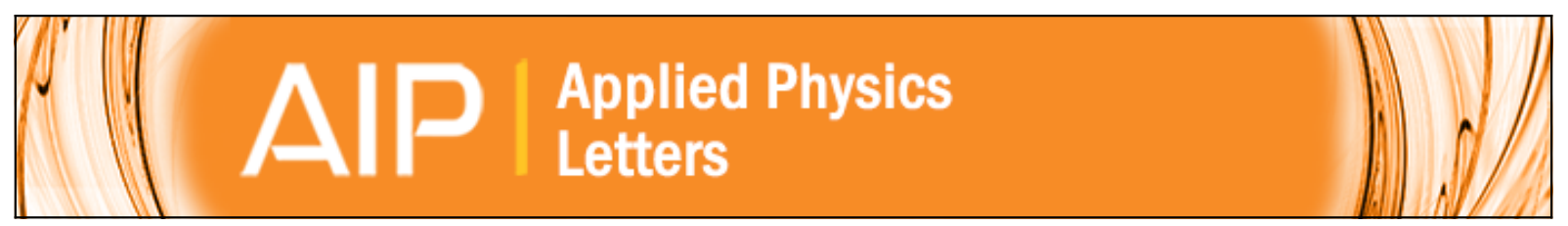

\title{
Oxidation of graphene in ozone under ultraviolet light
}

Y. C. Cheng, T. P. Kaloni, Z. Y. Zhu, and U. Schwingenschlögl

Citation: Applied Physics Letters 101, 073110 (2012); doi: 10.1063/1.4746261

View online: http://dx.doi.org/10.1063/1.4746261

View Table of Contents: http://scitation.aip.org/content/aip/journal/apl/101/7?ver=pdfcov

Published by the AIP Publishing

MULTIPHYSICS

SIMULATION

SIFECTRUM

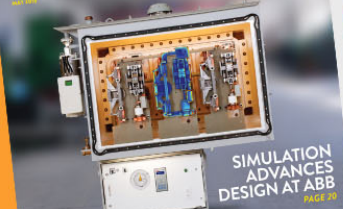

FREE Multiphysics Simulation e-Magazine
DOWNLOAD TODAY » 


\title{
Oxidation of graphene in ozone under ultraviolet light
}

\author{
Y. C. Cheng, T. P. Kaloni, Z. Y. Zhu, and U. Schwingenschlögl ${ }^{\text {a) }}$ \\ PSE Division, KAUST, Thuwal 23955-6900, Kingdom of Saudi Arabia
}

(Received 3 July 2012; accepted 2 August 2012; published online 17 August 2012)

\begin{abstract}
Based on first-principles calculations, the process of graphene oxidation in ozone under ultraviolet light is explored by studying the effects of $\mathrm{O}\left({ }^{3} \mathrm{P}\right)$ on the structural and electronic properties of a graphene sheet. On initial absorption of $\mathrm{O}\left({ }^{3} \mathrm{P}\right)$, an epoxy group is formed on the graphene sheet and the Dirac cone is shifted away from the Brillouin zone corners without carrier doping. When the oxidation progresses, an adjacent $\mathrm{C}-\mathrm{C}$ bond is broken, a vacancy is formed, and $\mathrm{CO}$ and/or $\mathrm{CO}_{2}$ molecules are released. (c) 2012 American Institute of Physics.

[http://dx.doi.org/10.1063/1.4746261]
\end{abstract}

Graphene is a planar $\mathrm{sp}^{2}$-bonded carbon material. Due to great potential in electronics application, it has attracted much attention since it was first fabricated in 2004. ${ }^{1}$ Driven by fundamental interest and potential applications, but also as an example for chemical functionalization, oxidation of graphene has been studied intensively. ${ }^{2-4}$ However, due to the amorphous nature of graphene oxide generated by the chemical fabrication method, understanding the atomic structure and its effects on the oxidation process is still a big challenge. Recently, various studies have dealt with the oxidation of graphene by ozone and $\mathrm{O}$ ions. ${ }^{5-14}$ In an ozone generator, $\mathrm{C}$ atoms are removed from the graphene lattice and vacancies are formed. The morphology of exposed singlelayer and multilayer graphene samples reflects a highly disordered nature, which varies with the thickness. ${ }^{5}$ After ozone generation by ultraviolet light, ozone molecules react with graphene to produce $\mathrm{O}$-containing groups and induce $p$-type doping. ${ }^{8}$ Theoretically, it has been predicted that $\mathrm{O}$ atoms lined up on pristine graphene form a stable unzipped structure, in which an epoxy group breaks the lower $\mathrm{sp}^{2}$ bond. ${ }^{9,14}$ In addition, the fact that the energy gap can be tuned in a wide range from 0 to $4 \mathrm{eV}$ demonstrates that functionalization of graphene by oxidation has serious effects on the electronic states. ${ }^{10,13}$ However, there exists little knowledge about the oxidation process itself.

In this work, we will study the initial steps of the oxidation process of graphene and the corresponding evolution of the structural and electronic properties by first principles calculations. We argue that the absorption of $\mathrm{O}\left({ }^{3} \mathrm{P}\right)$ is energetically favorable as compared to that of an ozone molecule $\left(\mathrm{O}_{3}\right)$. The initial absorption of $\mathrm{O}\left({ }^{3} \mathrm{P}\right)$ results in the formation of epoxy groups on the graphene. These groups induce a shift of the Dirac cone in the Brillouin zone as well as a pseudogap in the electronic density of states. In the further oxidation process $\mathrm{O}\left({ }^{3} \mathrm{P}\right)$ is absorbed next to the previously created defect, which leads to the breaking of $\mathrm{C}-\mathrm{C}$ bonds and generation of vacancies (holes). When the holes grow, $\mathrm{CO}$ and/or $\mathrm{CO}_{2}$ molecules are released. Therefore, the oxidation of graphene by ozone under ultraviolet light cannot be reverted.

Our calculations employ the generalized gradient approximation of the exchange correlation functional with norm-

a)udo.schwingenschlogl@kaust.edu.sa. Tel.: +966(0)544700080. conserving pseudopotentials, ${ }^{15}$ as implemented in the QuantumESPRESSO package. ${ }^{16}$ A cut-off energy of $544 \mathrm{eV}$ is used to achieve a high accuracy. A $5 \times 5$ supercell of pristine graphene is sufficiently large to accommodate the defects under investigation. This supercell comprises 50 atoms and has a lattice constant of $a=12.2 \AA$. To prevent interlayer interaction due to the periodic boundary conditions we add a vacuum slab of $20 \AA$. Structural optimization is carried out for all defect configurations up to an energy convergence of $10^{-7} \mathrm{eV}$ and a force convergence of $0.05 \mathrm{eV} / \AA$, employing a $6 \times 6 \times 1$ $\mathrm{k}$-mesh. Moreover, a Gaussian smearing of $41 \mathrm{meV}$ is used, which corresponds to an electronic temperature of $490 \mathrm{~K}$. The formation energy of $\mathrm{O}\left({ }^{3} \mathrm{P}\right)$ absorbed on graphene is evaluated by the relation $E_{f}=E_{s}+N \cdot E\left(\mathrm{CO} / \mathrm{CO}_{2}\right)-E_{i}-E(\mathrm{O})$, where $E_{i}$ and $E_{s}$ are the energies of the initial and oxidized graphene sheets. Furthermore, $E(\mathrm{O})$ and $E\left(\mathrm{CO} / \mathrm{CO}_{2}\right)$ denote the energies of $\mathrm{O}\left({ }^{3} \mathrm{P}\right)$ and $\mathrm{CO} / \mathrm{CO}_{2}$, and $N$ is the number of $\mathrm{CO} / \mathrm{CO}_{2}$ molecules.

There are two main methods for oxidizing graphene. The first is the application of an O plasma. ${ }^{12}$ The second, which is more common and simple, is the application of ozone as generated by ultraviolet light in atmosphere. ${ }^{5,8,11}$ In this case, $\mathrm{O}_{2}$ molecules absorb $185 \mathrm{~nm}$ photons, which have sufficient energy to break the molecular bond to form two $\mathrm{O}\left({ }^{3} \mathrm{P}\right)$ in the ground state: $\mathrm{O}_{2}+h v=2 \mathrm{O}\left({ }^{3} \mathrm{P}\right)$ and $\mathrm{O}\left({ }^{3} \mathrm{P}\right)+\mathrm{O}_{2}=\mathrm{O}_{3}$. In addition, the produced $\mathrm{O}_{3}$ can be destroyed by reaction with $\mathrm{O}\left({ }^{3} \mathrm{P}\right)$ as $\mathrm{O}_{3}+\mathrm{O}\left({ }^{3} \mathrm{P}\right)=2 \mathrm{O}_{2}$. This analysis shows that there are essentially three species, namely $\mathrm{O}\left({ }^{3} \mathrm{P}\right), \mathrm{O}_{2}$, and $\mathrm{O}_{3}$, involved in the oxidization process. By determining the optimized structures of graphene oxidized by these species, we find that $\mathrm{O}\left({ }^{3} \mathrm{P}\right)$ can bond effectively to the $\mathrm{C}$ atoms of the graphene, see Fig. 1(a). More specifically, it is absorbed on the bridge site of the $\mathrm{C}$ hexagons and forms an epoxy group. The formation energy is found to be $E_{f}=-5.6 \mathrm{eV} . \mathrm{O}_{3}$ turns out to be only physisorbed on graphene, in agreement with previous calculations. ${ }^{11}$ Therefore, we can restrict the following considerations to $\mathrm{O}\left({ }^{3} \mathrm{P}\right)$ absorption.

The electronic band structure of an epoxy group absorbed on graphene is shown in Fig. 2 along two Brillouin zone paths. The $\mathrm{K}$ point of the original graphene Brillouin zone (unit cell with $2 \mathrm{C}$ atoms) is folded to the $\mathrm{K}$ point of the Brillouin zone of the $5 \times 5$ supercell. ${ }^{17}$ According to the band structure in Fig. 2(a), we obtain a shift of the Dirac cone away from the K 
Top View

Side View

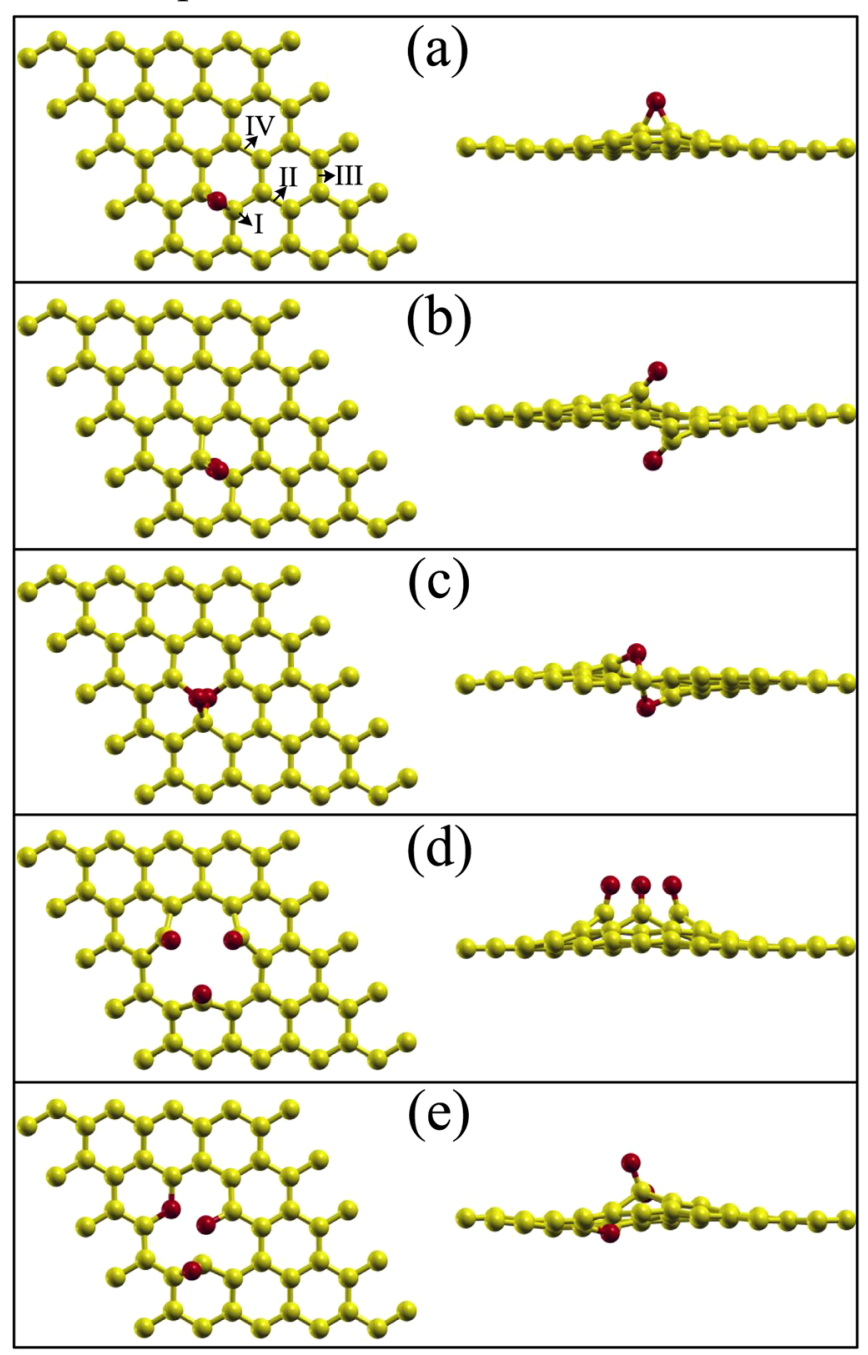

FIG. 1. Top and side views of relaxed structures of graphene oxide. (a) One $\mathrm{O}\left({ }^{3} \mathrm{P}\right)$ is absorbed on pristine graphene (bridge site). (b) An additional $\mathrm{O}\left({ }^{3} \mathrm{P}\right)$ breaks up the $\mathrm{C}-\mathrm{C}$ bond. (c) A third $\mathrm{O}\left({ }^{3} \mathrm{P}\right)$ absorbed on the defective site leads to the formation of $\mathrm{CO}$ and creation of a monovacancy. (d) The monovacancy is oxidized. (e) Further oxidization turns the monovacancy into a bivacancy. Red and yellow balls represent $\mathrm{O}$ and $\mathrm{C}$ atoms, respectively. point to the $\Gamma-\mathrm{K}_{1}$ line. In a previous study by Yan et al. ${ }^{10}$ (employing $4 \times 4$ supercell), it has been reported that the Dirac point shifts to the K-M line. This difference can be attributed to different Brillouin zone foldings. More importantly, we find no perfect Dirac cone but an energy gap of about $0.5 \mathrm{eV}$ with the valence band maximum and conduction band minimum along the $\mathrm{K}_{2}-\mathrm{M}_{2}$ line, Fig. 2(b). To further study this issue, we address the direct optical transition energy of the $\pi-\pi^{*}$ bands in the Brillouin zone of the $5 \times 5$ supercell in Fig. 2(c). A distinct shift of the Dirac cone is clearly visible. This shift as well as the band gap along the $\mathrm{K}_{2}-\mathrm{M}_{2}$ line can be attributed to a strong hybridization of the $\mathrm{O} 2 p$ orbitals with the extended $\pi$ bands of the graphene and the corresponding symmetry lowering induced by lattice distortions.

Raman spectroscopy of graphene can be used to obtain information about the number of layers, the charge carrier concentration, and the intrinsic/applied strain. ${ }^{18}$ The change of the electronic structure due to the absorption of an epoxy group likewise can be monitored. Although the spectrum contains contributions from all over the Brillouin zone, the main contribution is due to the $\mathrm{K}-\mathrm{K}^{\prime}$ valley. ${ }^{19}$ Since the shift of the Dirac cone induced by $\left.\mathrm{O}^{3} \mathrm{P}\right)$ absorption is similar to the shift induced by strain, ${ }^{20-22}$ the two-dimensional Raman band of graphene with an absorbed epoxy group is expected to be broadened.

In order to find the energetically favorable absorption site for the second $\mathrm{O}\left({ }^{3} \mathrm{P}\right)$ on graphene, we have investigated the four sites highlighted in Fig. 1(a) by labels I to IV. Our results show that the formation energy grows with the distance of the second from the first absorption site. Figure 1(b) presents the structure with the second $\mathrm{O}\left({ }^{3} \mathrm{P}\right)$ absorbed at site I, which yields the lowest formation energy of $E_{f}=-5.5 \mathrm{eV}$. It has been claimed that it is easy to form linear epoxy groups on graphene. ${ }^{14}$ However, there is no experimental or theoretical evidence for this claim. Our calculations show that the formation energy of the second $\mathrm{O}\left({ }^{3} \mathrm{P}\right)$ for absorption at the linear site IV is $1.8 \mathrm{eV}$ higher than for absorption at site I. The $\mathrm{O}\left({ }^{3} \mathrm{P}\right)$ bonds to the defective site near the first $\mathrm{O}$ atom, resulting in a breaking of the $\mathrm{C}-\mathrm{C}$ (a)

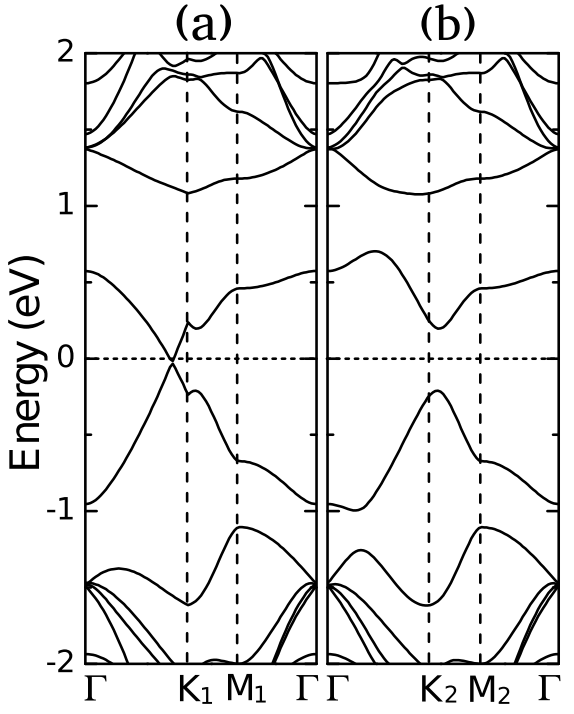

(c)

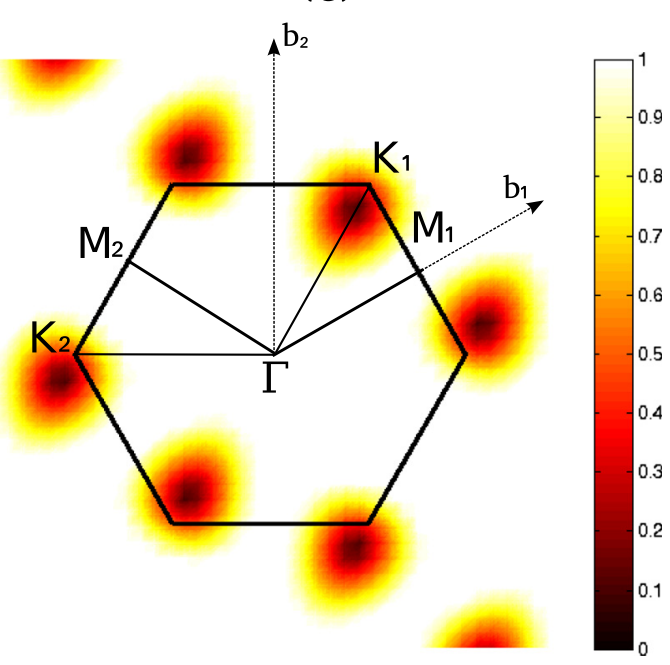

FIG. 2. (a and b) Band structures for one $\mathrm{O}\left({ }^{3} \mathrm{P}\right)$ absorbed on pristine graphene, see Fig. 1(a), along the paths $\Gamma-\mathrm{K}_{1}-\mathrm{M}_{1}-\Gamma$ and $\Gamma-\mathrm{K}_{2}-\mathrm{M}_{2}-\Gamma$. (c) Map of the direct optical transition energy of the $\pi-\pi^{*}$ bands in reciprocal space. 


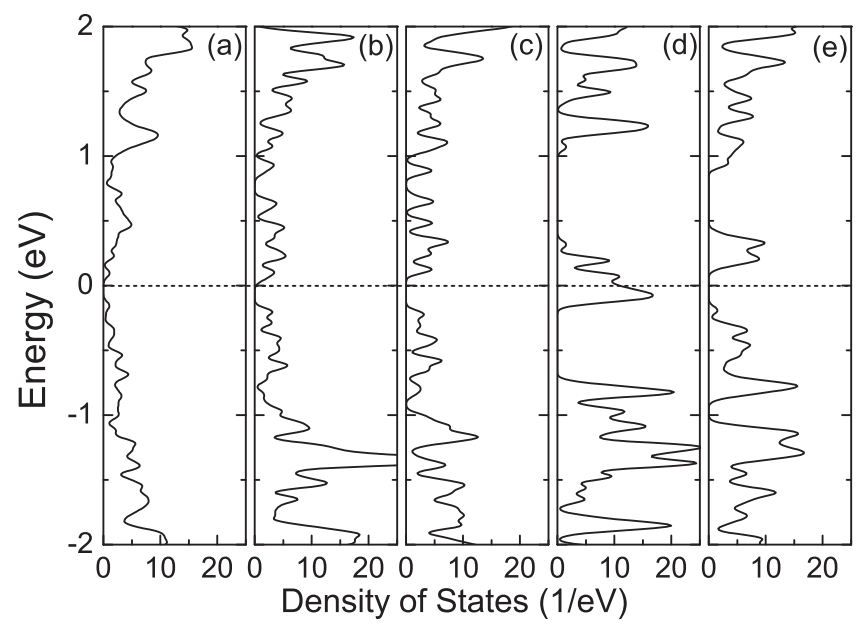

FIG. 3. Densities of states obtained for the structures defined in Fig. 1.

bond. Each $\mathrm{C}$ then bonds to one $\mathrm{O}$ atom (ketone group), as is demonstrated in Fig. 1(b). This finding is consistent with recent experimental data. ${ }^{12}$

Based on the above discussion, $\mathrm{O}\left({ }^{3} \mathrm{P}\right)$ prefers absorption at defective sites rather than at $\mathrm{C}$ atoms with $s p^{2}$ hybridization. Therefore, bonding of the third $\mathrm{O}\left({ }^{3} \mathrm{P}\right)$ leads to the formation of $\mathrm{CO}$ and creation of an oxidized monovacancy, see Fig. 1(c), according to the reaction $\mathrm{C}+\mathrm{O}\left({ }^{3} \mathrm{P}\right)=\mathrm{CO}$. The formation energy of this process is $E_{f}=-4.7 \mathrm{eV}$. In the oxidized monovacancy, the two $\mathrm{O}$ atoms form an ether group. Further oxidization causes each of the three inner $\mathrm{C}$ atoms at the monovacancy to be bonded to one $\mathrm{O}$ atom, see Fig. $1(\mathrm{~d})$. The ether group disappears and ketone groups are created, where the formation energy of this absorption step turns out to be $E_{f}=-4.1 \mathrm{eV}$. A detailed analysis of the resulting configuration can be found in Ref. 13. Continuation of the oxidation process can lead to the release of another $\mathrm{CO}$ molecule, see Fig. 1(e), coming along with a growth of the vacancy. The formation energy of this process is $E_{f}=-7.2 \mathrm{eV}$. However, it is also possible that two $\mathrm{O}\left({ }^{3} \mathrm{P}\right)$ are absorbed simultaneously at defective $\mathrm{C}$ sites in the configuration shown in Fig. 1(c). This leads to the release of a $\mathrm{CO}_{2}$ molecule (and the same enlargement of the vacancy reported before). The formation energy here amounts to $E_{f}=-15.6 \mathrm{eV}$.

The electronic densities of states obtained for the configurations in Figs. 1(a)-1(e) are shown in Fig. 3, which illustrates that a metallic behavior can be observed in graphene oxide. This is consistent with previous theoretical calculations. ${ }^{10,13}$ The pseudogaps visible in Figs. 3(a)-3(c) and 3(e) amount to about $0.2 \mathrm{eV}$, suggesting that the Dirac cone is maintained but a small band gap is opened for the atoms around the defect. The gap lies in the infrared region and therefore is difficult to be detected by photoluminescence spectroscopy. Previous photoluminescence experiments show that graphene with antidots generated by electron irradiation or oxygen plasma treatment is semiconducting because of a confinement effect. ${ }^{23}$ However, the sizes of the studied antidots (holes) are much larger than the vacancies in this work. In contrast to the previous cases, Fig. 3(d) is indicative of a metallic nature, where the density of states around the Fermi level is mainly due to the absorbed $\mathrm{O}$ atoms. Therefore, the different defects encountered in oxidized graphene during the oxidation process induce a complex variation of the electronic structure.

Epoxy groups are found to be the dominant species only in the low oxidation regime, which is consistent with the experimental situation. ${ }^{12}$ Further damage to the graphene lattice in the high oxidation regime is reflected by the appearance of $\mathrm{C}=\mathrm{O}$ groups, while the enlargement of the hole leads to a generation of $\mathrm{CO}$ and/or $\mathrm{CO}_{2}$ molecules. The formation of $\mathrm{C}=\mathrm{O}$ groups in graphene has been demonstrated experimentally by x-ray photoelectron spectroscopy. ${ }^{12}$ In the early oxidation stage graphene oxide can be reduced by $\mathrm{H}_{2}$ treatment, because the $\mathrm{C}-\mathrm{C}$ bonds of the graphene lattice are still intact. Moreover, the epoxy groups can diffuse on the graphene sheet subject to an energy barrier of about $0.8 \mathrm{eV} .{ }^{11}$ Based on this diffusion mechanism, two epoxy groups on the same side of the graphene sheet can merge to form an $\mathrm{O}_{2}$ molecule at high temperature, whereas an interaction of epoxy groups on opposite sides results in a breaking of the $\mathrm{C}-\mathrm{C}$ bond. Therefore, graphene oxide with low $\mathrm{O}$ concentration (mainly containing epoxy groups) can be reduced to pristine graphene by high temperature and/or hydrogen atmosphere. However, at a later oxidation stage a reduction is excluded as $\mathrm{C}-\mathrm{C}$ bonds are broken and/or $\mathrm{C}$ atoms have been released from the graphene sheet in form of $\mathrm{CO}$ and/or $\mathrm{CO}_{2}$ molecules.

In conclusion, we have studied the initial steps of the oxidation process of graphene by first principles calculations. We argue that the absorption of $\mathrm{O}\left({ }^{3} \mathrm{P}\right)$ is energetically favorable as compared to that of ozone molecules. Initial absorption of $\mathrm{O}\left({ }^{3} \mathrm{P}\right)$ leads to the formation of epoxy groups on graphene, which induce a shift of the Dirac cone in the Brillouin zone. In the following oxidation process, absorption of $\mathrm{O}\left({ }^{3} \mathrm{P}\right)$ is favorable at the previously created defective sites without $\mathrm{C} s p^{2}$ hybridization. Absorption at such sites induces a breaking of C-C bonds and consequently generates vacancies in the graphene sheet. Under further oxidation, the vacancies grow to bigger holes and $\mathrm{CO}$ and/or $\mathrm{CO}_{2}$ are released. Once $\mathrm{C}-\mathrm{C}$ bonds have been broken, the oxidation of graphene by ozone under ultraviolet light cannot be reverted.

${ }^{1}$ K. S. Novoselov, A. K. Geim, S. V. Morozov, D. Jiang, Y. Zhang, S. V. Dubonos, I. V. Grigorieva, and A. A. Frisov, Science 306, 666 (2004).

${ }^{2}$ J.-L. Li, K. Kudin, M. McAllister, R. Prudhomme, I. Aksay, and R. Car, Phys. Rev. Lett. 96, 176101 (2006).

${ }^{3}$ D. A. Dikin, S. Stankovich, E. J. Zimney, R. D. Piner, G. H. B. Dommett, G. Evmenenko, S. T. Nguyen, and R. S. Ruoff, Nature 448, 457 (2007).

${ }^{4}$ X. Wu, M. Sprinkle, X. Li, F. Ming, C. Berger, and W. A. de Heer, Phys. Rev. Lett. 101, 026801 (2008).

${ }^{5}$ H. Tao, J. Moser, F. Alzina, Q. Wang, and C. M. Sotomayor-Torres, J. Phys. Chem. 115, 18257 (2011).

${ }^{6}$ C. Gómez-Navarro, J. C. Meyer, R. S. Sundaram, A. Chuvilin, S. Kurasch, M. Burghard, K. Kern, and U. Kaiser, Nano Lett. 10, 1144 (2010).

${ }^{7}$ A. Sinitskii and J. M. Tour, J. Am. Chem. Soc. 132, 14730 (2010).

${ }^{8}$ S. Huh, J. Park, Y. S. Kim, K. S. Kim, B. H. Hong, and J.-M. Nam, ACS Nano 5, 9799 (2011).

${ }^{9}$ Z. Xu and K. Xue, Nanotechnology 21, 045704 (2010).

${ }^{10}$ J.-A. Yan and M. Chou, Phys. Rev. B 82, 125403 (2010).

${ }^{11}$ G. Lee, B. Lee, J. Kim, and K. Cho, J. Phys. Chem. C 113, 14225 (2009).

${ }^{12}$ R. Larciprete, P. Lacovig, S. Gardonio, A. Baraldi, and S. Lizzit, J. Phys. Chem. C 116, 9900 (2012).

${ }^{13}$ T. P. Kaloni, Y. C. Cheng, R. Faccio, and U. Schwingenschlögl, J. Mater. Chem. 21, 18284 (2011). 
${ }^{14}$ Z. Li, W. Zhang, Y. Luo, J. Yang, and J. G. Hou, J. Am. Chem. Soc. 131, 6320 (2009).

${ }^{15}$ D. R. Hamann, M. Schlüter, and C. Chiang, Phys. Rev. Lett. 43, 1494 (1979). ${ }^{16}$ P. Giannozzi, S. Baroni, N. Bonini, M. Calandra, R. Car, C. Cavazzoni, D. Ceresoli, G. L. Chiarotti, M. Cococcioni, I. Dabo, A. D. Corso, S. de Gironcoli, S. Fabris, G. Fratesi, R. Gebauer, U. Gerstmann, C. Gougoussis, A. Kokalj, M. Lazzeri, L. Martin-Samos, N. Marzari, F. Mauri, R. Mazzarello, S. Paolini, A. Pasquarello, L. Paulatto, C. Sbraccia, S. Scandolo, G. Sclauzero, A. P. Seitsonen, A. Smogunov, P. Umari, and R. M. Wentzcovitch, J. Phys.: Condens. Matter 21, 395502 (2009).

${ }^{17}$ Y. C. Cheng, T. P. Kaloni, G. S. Huang, and U. Schwingenschlögl, Appl. Phys. Lett. 99, 053117 (2011).
${ }^{18}$ A. C. Ferrari, J. C. Meyer, V. Scardaci, C. Casiraghi, M. Lazzeri, F. Mauri, S. Piscanec, D. Jiang, K. S. Novoselov, S. Roth, and A. K. Giem, Phys. Rev. Lett. 97, 187401 (2006).

${ }^{19}$ R. Narula and S. Reich, Phys. Rev. B 78, 165422 (2008).

${ }^{20}$ M. Mohr, K. Papagelis, J. Maultzsch, and C. Thomsen, Phys. Rev. B 80, 205410 (2009).

${ }^{21}$ Y. C. Cheng, Z. Y. Zhu, G. S. Huang, and U. Schwingenschlögl, Phys. Rev. B 83, 115449 (2011).

${ }^{22}$ D. Yoon, Y.-W. Son, and H. Cheong, Phys. Rev. Lett. 106, 155502 (2011).

${ }^{23}$ S. Heydrich, M. Hirmer, C. Preis, T. Korn, J. Eroms, D. Weiss, and C. Schüler, Appl. Phys. Lett. 97, 043113 (2010). 